\title{
STRUCTURAL HEALTH MONITORING APPROACH TO THE AEROSPACE STRUCTURES
}

\author{
Krzysztof Dragan \\ Air Force Institute of Technology, Warsaw, Poland
}

\begin{abstract}
In the article only selected information regarding the SHM applications has been presented. These applications are associated with damage detection and damage growth monitoring. That approach has got crucial influence on the aircraft maintenance procedures. SHM ensures higher reliability and safety of the structures as well as repair monitoring. Right now in the AFIT, there is being conducted research work focused on the application of the SHM system for the integration with the aerospace structures for periodical and on-line monitoring.
\end{abstract}

\section{INTRODUCTION}

In recent years there has been observed an enormous growth of interest in continuous and periodical condition monitoring of civil and aerospace structures with the use of Structural Health Monitoring techniques (called SHM) [1,2]. Such systems use 'physics' of NDT for data acquisition with the use of so called "smart layers" $[3,4]$. These "smart layers" are based on sensor networks distributed in the structure of the object under monitoring. Such an approach enables condition monitoring with limited human interaction. In this article, a brief description of such techniques for the aerospace application will be delivered. Techniques such as the guided waves with the use of PZT application as well as CVM ${ }^{\mathrm{TM}}$ (Comparative Vacuum Monitoring), will be described [5]. Moreover, a few examples of the monitoring activities conducted by the AFIT will be presented.

\section{METHODOLOGY OF CRACK GROWTH ANALYSIS}

One of the important examples of the monitoring necessity is rotorcrafts. According to the report by the Australian Transportation Board [6]: from 1985 to 2003 there were 776 accidents with the increased trend (36 accidents in 1985 to 43 in 2001).

One of the dramatic examples of the monitoring necessity was the accident of the military helicopter in France where 8 people were killed in the flight accident caused by the main rotor blade separation [7].

In the main rotor blade of the helicopters used in the Armed Forces as well as in more popular smaller rotorcrafts, there occur fatigue cycle phenomena which may lead to critical failure [8]. Monitoring such failure modes is crucial from the safety and durability of the structure point of view [9]. It is possible to describe the critical damage areas, which is very important from the maintenance point of view [8].

Monitoring such areas may be performed with the use of the classic NDT approach [10]. Modern maintenance philosophies require a more advanced approach to structural integrity monitoring such as the multimode NDE approach. 
The failure modes crucial from the maintenance point of view are: SCC [1], fatigue cracks and material defects [8]. In picture 1, the failure mode scenario for the main rotor blade has been presented.
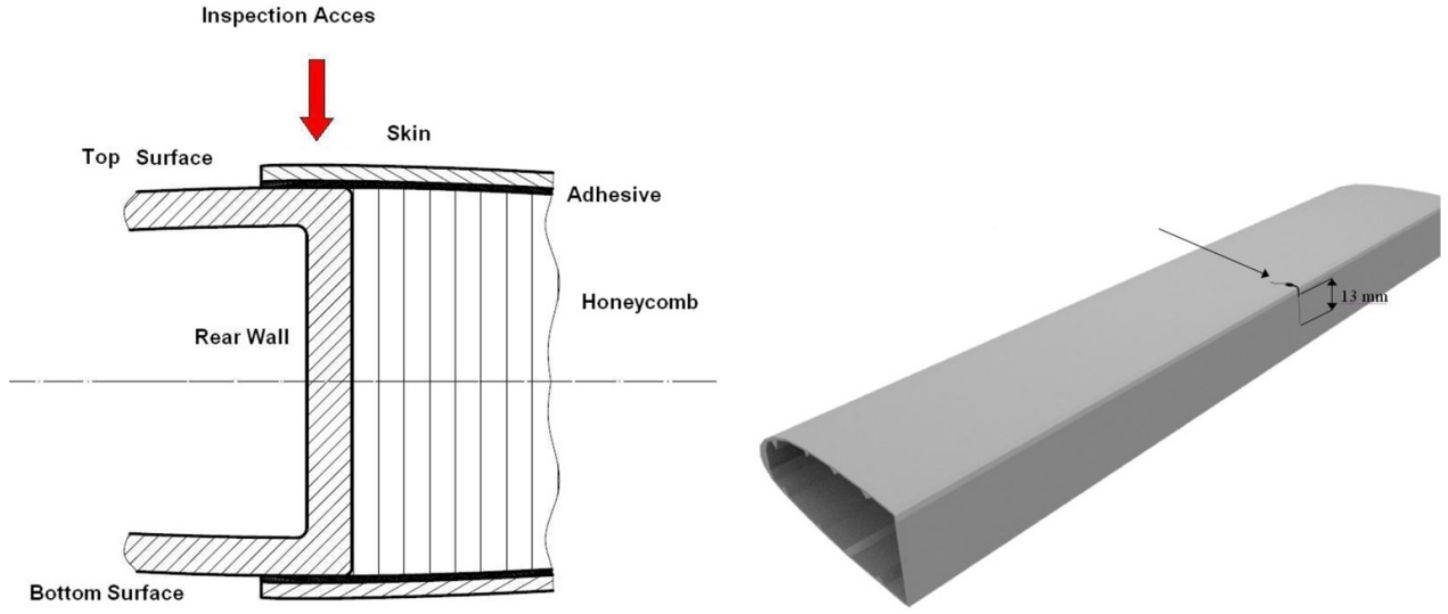

Fig. 1. Geometry for inspection

The structural assessment of the rear web of the main rotor blade is more difficult not only from the accessibility point of view. Other issues are: the height to width ratio of the inspected element as well as lack of signal transmission between the skin and the spar (Pic. 2). During R\&D work conducted in the Institute (AFIT) for the structure assessment, conventional NDT methods have been used (ultrasonic and eddy current). But these techniques do not allow for rear web inspection. Because of that, monitoring techniques which use the embedded sensors have been applied. For that purpose the CVM ${ }^{\mathrm{TM}}$ and PZT sensors were used.
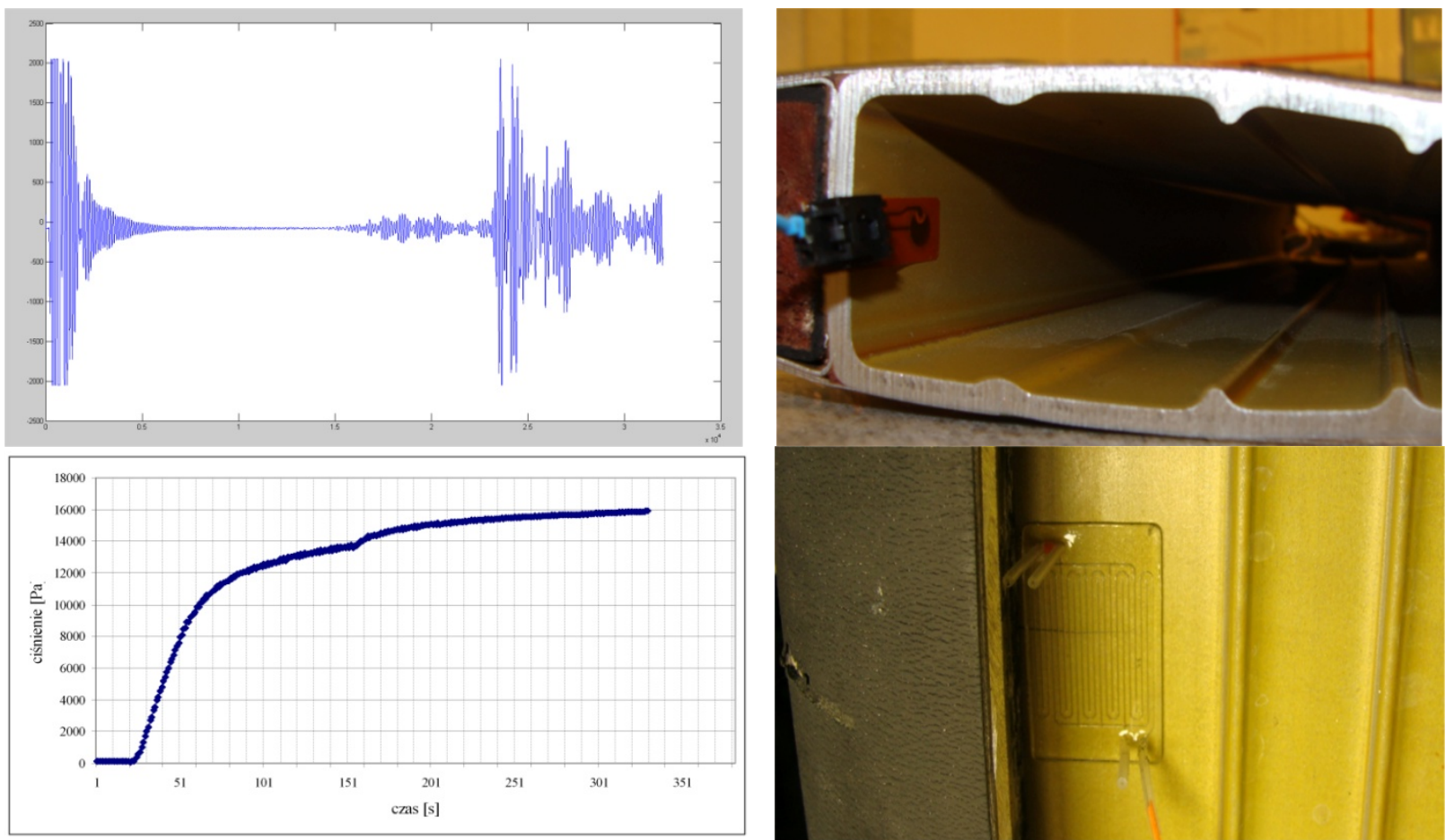

Fig. 2. Blade monitoring of the rear wall with the use of PZT and CVMTM and signal from the sensors 
For the blade monitoring, the PZT sensors were used. These sensors enable elastic waves generation in the blade structure. For such geometry, the Rayleigh - Lamb waves are generated. Because of thin wall geometry, $\mathrm{A}$ and $\mathrm{S}$ (antysymmetric and symmetric) wave modes are generated. The Rayleigh - Lamb waves may be described with the relation [11]:

$$
\frac{\tanh (\mathrm{qd})}{\tan (p d)}=\left[\frac{4 k^{2} p q}{\left(q^{2}-k^{2}\right)}\right]^{ \pm m}
$$

where:

$d$ - material thickness;

$k$ - wave number: $k=\omega / V_{f}$ (phase velocity) $\left(k_{t}, k_{1}\right.$ - wave numbers for compressional and shear wave); $m$ - exponent ( +1 symmetric modes, -1 antisymmetric modes);

$p^{2}=\left(k_{t}^{2}-k^{2}\right) ; \quad q^{2}=\left(k_{s}^{2}-k^{2}\right)$.

Based on the wave propagation analysis as well as the phenomena connected with reflection and mode conversion, the signal analysis is being performed. Such analysis enables damage detection and damage description. The advantages of this method are following: possibility of signal transmission for further distances, sensitivity to changes in the structure integrity.

Limitations are connected with the interpretation of the data and the necessity for the signal modeling. Also, difficulties connected with boundary interaction of the wave and mode separation may appear. From that point of view, the necessity of the modeling must be taken into consideration.

Another technique called CVM ${ }^{\mathrm{TM}}$ is connected with the crack monitoring. This technique is simpler then wave generation. Monitoring is based on local crack detection. Sensors consist of multiple measurement galleries. There are two channels in each sensor (connected to vacuum generator and ambient pressure). Damage localization is connected with the vacuum generation in the measurement galleries. The principle of work has been presented in Fig. 3. Depending on the crack length, crack orientation and sensor shape, different pressure variations indicate crack propagation under the sensor, which means there is a possibility for crack length estimation.
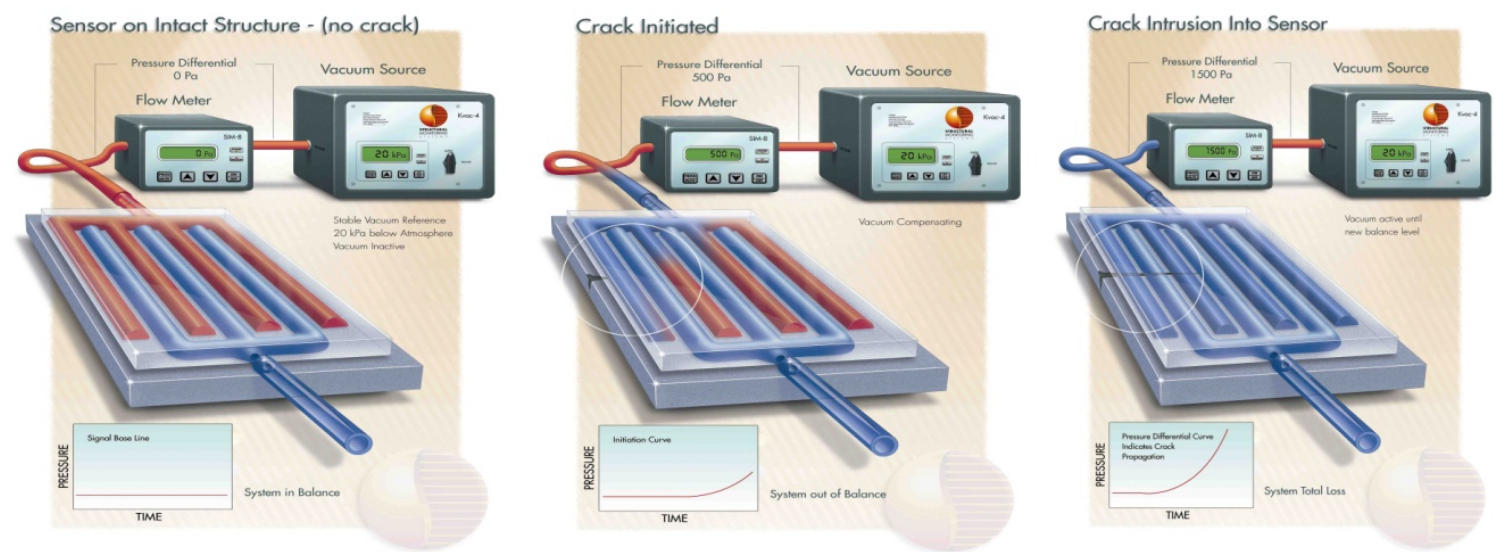

Fig. 3 Principle of work of the CMM system

The main advantages of the method are the following: possibility of fast and indirect measurement, lack of electrical cabling, on-line monitoring capability (which is very important from the point of view of aerospace structure monitoring). The disadvantages are connected with the necessity of structure monitoring in ,hot - spot” locations and for that reason global monitoring is not possible. 


\subsection{CENTRIFUGE FOR MEDICAL TEST OF PILOTS MONITORING}

The structure of the centrifuge is similar to the structures used in the aerospace solutions. In that case it is possible to determine: spars and ribs, skin and fasteners. Also, there is a similarity connected with the materials and geometry. Based on that, it is possible to use monitoring techniques similar to those used in aerospace. One of the difficulties for the inspection is button head fasteners. For such structures, the classic approach with the use of NDE techniques may not be efficient. Based on the AFIT experience of conducting inspections, the critical "hot spots" have been determined.

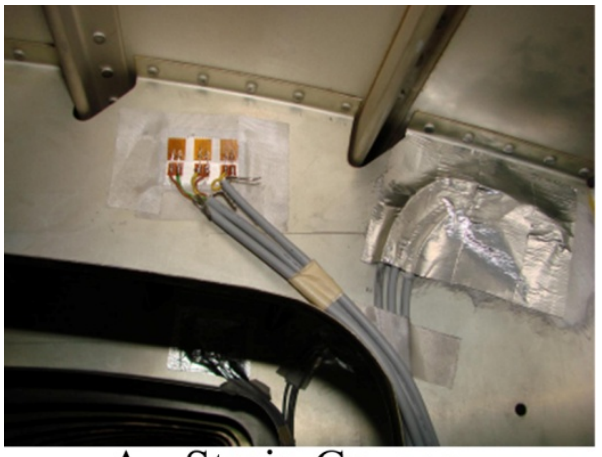

A - Strain Gauges

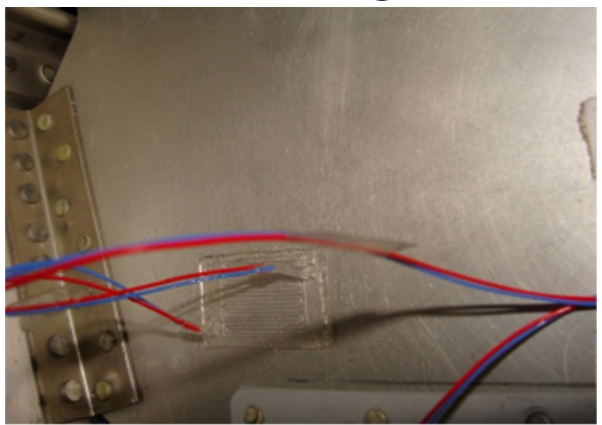

C - CVM sensors

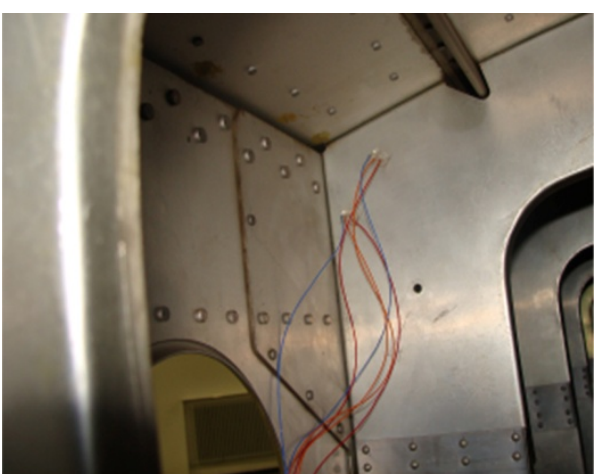

B - CVM sensors

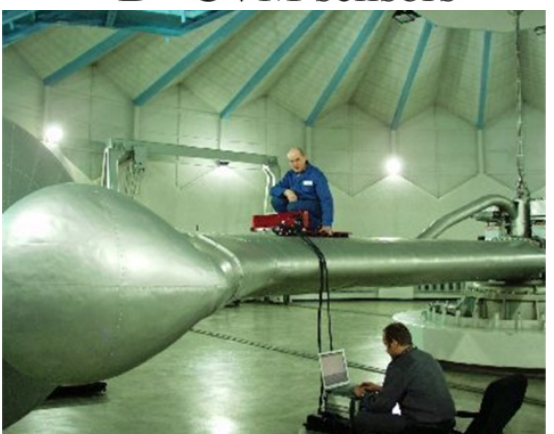

D - NDT

Fig. 4 Monitoring areas of the strain gauges

In the described areas, the $\mathrm{CVM}^{\mathrm{TM}}$ sensors as well as strain gauges were applied. At present, the application is used for periodical monitoring. Right now, there are being determined work packages which may enable system integration with the centrifuge data acquisition system as well as works connected with data comparison from CVM sensors with the NDE inspections. 


\section{REFERENCES}

[1] (1997). Aging of U.S. Air Force Aircraft. USA, Washington D.C.: National Academy Press. (NMAB-488-2)

[2] Dragan, K. (2006). In-service Flaw Detection and Quantification on the MiG-29 Composite Vertical Tail Skin. In the $9^{\text {th }}$ European Conference on Non-Destructive Testing, Berlin, 25-29 Września 2006.

[3] Rücker, W. (2006). SAMCO Final Report 2006, Guideline for Structural Health Monitoring. Berlin: Federal Institute of Materials Research and Testing (BAM).

[4] Giurgiutiu, V. (2008). Structural Heath Monitoring and NDE - An Air Force Office of Scienific Research Structural Mechanics Program Perspective. In the $4^{\text {th }}$ European Workshop on SHM, Kraków, July 2-4 2008, (35 - 39).

[5] Speckmann, H., \& Henrich, R. (2004). Structural Health Monitoring (SHM) - Overview On Technologies Under Development. In the $16^{\text {th }}$ WCNDT 2004 - World Conference on NDT, Canada: Montreal, August 30 - Sep 32004.

[6] Light Utility Helicopter Safety in Australia, RESEARCH PAPER BE04/73, Australian Transport Safety Bureau (ATSB), June 2004.

[7] Web page http://www.ntsb.gov/publictn/1996/SIR9603.pdf

[8] Shaniavski, A., A. (2004). Scale levels for fatigue fracture mechanisms of in-service crack growth in longerons of helicopter rotor blades. International Journal of Fracture. 128 (1), p. 17-24. DOI: 10.1023/B:FRAC.0000040960.52699.b9.

[9] Chronkite, J., D. (1993). Practical Application of Health and Usage Monitoring (HUMS) to Helicopter Rotor, Engine and Drive System. In the $49^{\text {th }}$ Forum of the American Helicopter Society. Alexandria, VA: American Helicopter Society.

[10]Dragan, K. (2009). NDE activities connected with Service Life Extension of Main Rotor Blades of Polish Military Helicopters. In the $12^{\text {th }}$ Aging Aircraft Conference, Kansas City, USA, 4-7 May 2009.

[11]Graff, K., F. (1991). Waves motion in elastic solids. New York: Dover Publications, Inc. 